\title{
Postprandial metabolic heterogeneity in men with primary dyslipidaemia
}

Antonios N. Pavlidis ${ }^{1}$, Genovefa D. Kolovou ${ }^{1}$, Katherine K. Anagnostopoulou ${ }^{2}$, Petros C. Petrou ${ }^{1}$, Dennis V. Cokkinos ${ }^{1}$

${ }^{1} 1^{\text {st }}$ Cardiology Department, Onassis Cardiac Surgery Centre, Athens, Greece 2Molecular Immunology Laboratory, Onassis Cardiac Surgery Center, Athens, Greece

Submitted: 22 July 2010

Accepted: 19 August 2010

Arch Med Sci 2010; 6, 6: 879-886

DOI: 10.5114/aoms.2010.19295

Copyright (c) 2010 Termedia \& Banach
Corresponding author:

Antonios N. Pavlidis, MD, PhD

P. Tsaldari $10 \mathrm{~A} 1$

Kifissia, 14561

Athens, Greece

Phone: +306947807977

Fax: +302106646902

E-mail: antonispav@yahoo.com

\begin{abstract}
Introduction: Familial combined hyperlipidaemia (FCH) and familial hypercholesterolaemia ( $\mathrm{FH}$ ) have been strongly linked to premature coronary artery disease. Postprandial hypertriglyceridaemia is also associated with atherosclerotic disease. We evaluated the postprandial lipaemia in men with $\mathrm{FCH}$ and $\mathrm{FH}$ and compared them to a group of healthy men.

Material and methods: The study population consisted of 83 men: $34 \mathrm{FCH}, 29 \mathrm{FH}$ and 20 healthy. The $\mathrm{FCH}$ and $\mathrm{FH}$ groups were further divided into five subgroups, according to their lipid phenotype: FCH-IIA $(n=13), \mathrm{FCH}-\mathrm{IIB}(n=10), \mathrm{FCH}-\mathrm{IV}$ $(n=11)$, FH-IIA $(n=21)$ and FH-IIB $(n=8)$. Postprandial lipaemia was evaluated by the areas under the curve for triglyceride (TG) concentrations (TG-AUC).

Results: The TG levels after oral fat tolerance test were significantly higher in $\mathrm{FCH}$, compared to $\mathrm{FH}$ and healthy groups (TG-AUC in $\mathrm{mg} / \mathrm{dl} / \mathrm{h} ; 2678 \pm 1415 \mathrm{vs}$. $1503 \pm 1147$ and $1011 \pm 652$ respectively, $p<0.001$ ). The postprandial response was higher in FCH-IV and FCH-IIB, compared to FCH-IIA (TG-AUC in mg/dl/h; $3220 \pm 824$ or $3409 \pm 770$ vs. $1863 \pm 577$ respectively, $p<0.001$, for both comparisons). The FCH-IIA group showed higher postprandial TG levels when compared to FH-IIA (TG-AUC in mg/dl/h; $1863 \pm 577$ vs. $1374 \pm 428$ respectively, $p=0.008$ ). There were no significant differences between FH-IIB and FCH-IIB subgroups. There was a significant correlation $(r=0.907, p<0.001)$ between the postprandial TG-AUC and fasting TG levels in all FCH subjects.

Conclusions: All phenotypes of FCH and the FH IIB phenotype demonstrate an exaggerated postprandial response that could partially contribute to the high cardiovascular risk. These patients should be identified and treated early with the appropriate hypolipidaemic agents.
\end{abstract}

Key words: postprandial lipaemia, familial combined hyperlipidaemia, familial hypercholesterolaemia

\section{Introduction}

The majority of epidemiological studies (Framingham, Prospective Cardiovascular Münster [PROCAM], Multiple Risk Factor Intervention Trial [MRFIT]) come to conclusions regarding triglycerides (TG) as a cardiovascular (CV) risk factor, based on fasting TG levels [1-3]. Lately, studies have suggested that non-fasting TG may predict CV events similarly or even better, compared to fasting TG, and have the practical advantage that patients do not need to fast [4]. Also, it is accepted that atherosclerosis is a postprandial phenomenon; thus investigating the fate of lipoproteins 
after the administration of a fat meal may be very useful in certain subjects $[5,6]$.

Familial combined hyperlipidaemia (FCH) accounts for approximately 100,000 myocardial infarctions per year in the United States and the European Union [7]. FCH is characterised by intraindividual and intrafamilial variability of the lipid profile, expressed as periodic increase of TG and/or total cholesterol (TC) levels [8].

Familial hypercholesterolaemia $(\mathrm{FH})$ is an autosomal, co-dominant, monogenic disorder of lipoprotein metabolism, characterised by very high levels of low density lipoprotein (LDL), tendon xanthomas and increased risk of premature atherosclerosis.

Postprandial lipaemia is a physiological polygenic metabolic process, following ingestion of dietary fat, mainly characterised by a marked increase in TG rich lipoprotein levels, such as chylomicrons $(\mathrm{CM})$, very low density lipoproteins (VLDL) and their remnants [5].

We evaluated the postprandial TG response after a fat tolerance meal in men with $\mathrm{FCH}$ and $\mathrm{FH}$ and a group of healthy subjects.

\section{Material and methods}

\section{Participants}

The independent ethics committee of the Onassis Cardiac Surgery Center in Athens approved the study protocol and all participants gave informed written consent. The study population consisted of 83 Greek men who where referred to our Lipid Clinic. Only patients without hypolipidaemic treatment were eligible to enter the study. Heavy drinking (more than 3 units/day), thyroid, liver or renal disorders and professional sport activity were exclusion criteria.

This study complies with the Declaration of Helsinki.

\section{Study groups}

The study population was divided into three main groups:

1. The FH group $(n=29)$, median age 34 (16). The diagnosis of $\mathrm{FH}$ was based on the widely accepted Simon Broome diagnostic criteria [9]: a) TC > $290 \mathrm{mg} / \mathrm{dl}$, LDL cholesterol $>190 \mathrm{mg} / \mathrm{dl}$, b) tendinous xanthomata in the patient or a first degree relative, c) family history of myocardial infarction in a first degree relative age $<60$ years or in a second degree relative age $<50$ years and d) family history of raised TC $>290 \mathrm{mg} / \mathrm{dl}$ in a first or second degree relative.

2. The FCH group $(n=34)$, median age 47 (14). The FCH status was diagnosed according to widely used diagnostic criteria [10, 11]: a) TC and/or TG levels $>90^{\text {th }}$ percentile, adjusted for age and gender, on the basis of the PROCAM study [2], b) primary variability of the lipid phenotype in the patient and/or one member of the family, c) plasma apoB concentrations $>120 \mathrm{mg} / \mathrm{dl}$, d) family history of myocardial infarction in a first degree relative age $<60$ years, or in a second degree relative age $<50$ years and e) absence of xanthomas.

3. The control group $(n=20)$, median age 46 (11). There was no previous medical history or family history of premature atherosclerosis, diabetes mellitus (DM), hypertension or dyslipidaemia. None of them was receiving any treatment. Three of the subjects were smokers.

\section{Study subgroups}

$\mathrm{FCH}$ and $\mathrm{FH}$ groups were further divided into subgroups, according to the Fredrickson lipid phenotype, by using the Lipid Research Clinic reference values [12]. Type IIA (hypercholesterolaemic phenotype) defined as $\mathrm{TC} \geq 95^{\text {th }}$ percentile, type IIB (mixed, hypercholesterolaemic and hypertriglyceridaemic phenotype) as both TG and TC $\geq 90^{\text {th }}$ percentile and type IV (hypertriglyceridaemic phenotype) as TG $\geq 95^{\text {th }}$ percentile.

FH subjects were divided into two subgroups, $\mathrm{FH}-\mathrm{IIA}(n=21)$ and FH-IIB $(n=8)$, and FCH subjects into three subgroups, FCH-IIA $(n=13)$, FCH-IIB $(n=10)$ and FCH-IV $(n=11)$. Postprandial TG response was compared among the three different $\mathrm{FCH}$ subgroups, as well as between $\mathrm{FH}$ and $\mathrm{FCH}$ subgroups with the same lipid phenotype (FH-IIA vs. FCH-IIA and FH-IIB vs. FCH-IIB).

\section{Fat tolerance test}

All subjects underwent a standardized oral fat tolerance test (FTT) following a $12 \mathrm{~h}$ overnight fast. Participants were forbidden to eat for $8 \mathrm{~h}$ after consumption of the meal, but were allowed free access to water. Each participant was given a meal containing $75 \mathrm{~g}$ fat $/ \mathrm{m}^{2}$ of body surface area, as previously described [13]. The meal provided $83.5 \%$ of energy from fat, $14.0 \%$ from carbohydrates and $2.5 \%$ from protein and was given in a dose based on the patient's body surface area (350 $\mathrm{g}$ for $2 \mathrm{~m}^{2}$ ). It was consumed within 20 minutes and TG levels were measured at 0, 2, 4, 6 and 8 hours (h). TC and high density lipoprotein (HDL) levels were only measured in the fasting state, since it is has been shown that their levels do not present significant alterations postprandially $[14,15]$.

\section{Plasma lipid, lipoprotein and apolipoprotein analysis}

Plasma TC, TG and HDL cholesterol levels were measured using enzymatic colorimetric methods, 
on a Roche Integra Biochemical analyser, with commercially available kits (Roche Diagnostics $\mathrm{GmbH}$, Mannheim, Germany). The serum LDL cholesterol levels were calculated using the Friedewald formula [16], only in subjects with TG levels $<400 \mathrm{mg} / \mathrm{dl}$. Three FCH patients had TG $>400 \mathrm{mg} / \mathrm{dl}$, so LDL was not calculated. ApoA, apoB and $L p(a)$ were measured by nephelometry (Nephelometer: BN-100, Behring, Germany). All samples were analysed within $24 \mathrm{~h}$.

\section{Glucose, insulin and insulin resistance analysis}

Blood glucose was measured by the hexokinase method with a Dade Behring reagent on a Dimension (Dade Behring, Liederbach, Germany) instrument and blood insulin with the IMX ABBOTT Diagnostics instrument. Whole-body insulin resistance was assessed with the HOMA-IR formula (fast glucose $\times$ fast insulin/22.5) [17]. All samples were analysed within $24 \mathrm{~h}$.

\section{Statistical methods}

All data were collected in an MS-Excel 2007 spreadsheet and analysed using SPSS 14.0 (SPSS Inc, Chicago, Illinois, USA) for Windows. Categorical variables are presented as percentages. Values of numerical characteristics were tested for normality and are presented as mean value $( \pm S D)$ if normally distributed, and median $( \pm I Q R)$ if not distributed normally. Area under the curve (AUC) for serial measurements of TG levels at baseline and after the fatty meal was calculated using the trapezoid rule. An ANOVA or Kruskal-Wallis $\mathrm{H}$ test with a Bonferroni correction (whichever appropriate) was performed for three group comparisons. The t-test for independent samples or the Mann-Whitney $U$ test was used for the comparison of numerical values between different groups, where appropriate. Chi square test was performed for comparison of categorical values between groups. Spearman's rank correlation coefficient was used as a measure of association between variables in order to reveal any correlation between values not normally distributed. The level of significance was set at $p \leq 0.05$.

\section{Results}

The fat loading test was ingested and tolerated well by all subjects. The amount of fatty meal ingested was 341 (19) g, 341 (35) g and 357 (41) g for controls, $\mathrm{FH}$ and $\mathrm{FCH}$ groups respectively.

\section{Baseline characteristics of the three main groups}

Clinical characteristics of the three main groups (controls, FH and FCH) are shown in Table I.
FCH subjects had higher body mass index (BMI) and waist values, compared to controls and $\mathrm{FH}$. As expected, DM, insulin resistance and hypertension were also more prevalent in the $\mathrm{FCH}$ group, compared to others. Fasting $\mathrm{TG}\left(\mathrm{TG}_{0}\right)$ levels were significantly higher in the $\mathrm{FCH}$ group, compared to controls and $\mathrm{FH}$, and were also higher in $\mathrm{FH}$ compared to controls.

\section{Baseline characteristics of different subgroups}

Clinical characteristics of the five subgroups (FH-IIA, FH-IIB, FCH-IIA, FCH-IIB, and FCH-IV) are summarised in Table II.

The FCH-IIA group had higher BMI and waist values, compared to FH-IIA. DM and hypertension were more prevalent in $\mathrm{FCH}-\mathrm{IIA}$, compared to FH-IIA. FCH-IIB and FCH-IV had higher $\mathrm{TG}_{0}$ levels compared to FCH-IIA $(p<0.001)$. Metabolic syndrome was more prevalent in $\mathrm{FCH}-\mathrm{IV}$, compared to $\mathrm{FCH}-\mathrm{IIB}(p=0.04)$, as well as in FCH-IIA, compared to $\mathrm{FH}-\mathrm{IIA}(p=0.001)$. No differences were observed in $\mathrm{TG}_{0}$ levels between $\mathrm{FH}$ and $\mathrm{FCH}$ subgroups with the same Fredrickson phenotype.

\section{Postprandial characteristics of the three main groups}

FCH showed significantly increased postprandial TG levels, at all hours, compared to controls and $\mathrm{FH}$. FH showed significantly increased postprandial TG levels, at all hours, compared to controls.

\section{Postprandial characteristics of different subgroups}

Comparison of postprandial TG levels between the three different $\mathrm{FCH}$ subgroups is shown in Figure 1.

FCH-IV and FCH-IIB showed higher area under the curve for TG concentrations (TG-AUC), compared to FCH-IIA ( $p<0.001)$. TG-AUC in FCH-IIA was significantly higher, compared to $\mathrm{FH}$-IIA ( $p=0.008)$. No significant postprandial differences were observed between FCH-IV and FCH-IIB or between FH-IIB and FCH-IIB subgroups.

\section{Correlations}

There was a significant correlation $(r=0.907$, $p<0.001$ ) between the postprandial TG-AUC values and $\mathrm{TG}_{0}$ in all $\mathrm{FCH}$ subjects. There were no significant correlations in the control group. A correlation between fasting TG levels and postprandial lipaemia in men with $\mathrm{FH}$ has already been shown [18].

\section{Discussion}

We evaluated the response to FTT in the two most common hereditary dyslipidaemias and 
Table I. Clinical characteristics of the three main groups

\begin{tabular}{|c|c|c|c|c|}
\hline Characteristics & Controls $(n=20)$ & $\mathrm{FH}(n=29)$ & $\mathrm{FCH}(n=34)$ & $p$ values \\
\hline Age [years] & $46(11)$ & $34(16)$ & $47(14)$ & A: <0.001; B: NS; C: 0.001 \\
\hline Body mass index $\left[\mathrm{kg} / \mathrm{m}^{2}\right]$ & $26(3)$ & $25(4)$ & $28(4)$ & A: NS; B: 0.002; C: $<0.001$ \\
\hline Waist $[\mathrm{cm}]$ & $94(10)$ & $95(12)$ & $103(12)$ & A: NS; B: 0.007; C: 0.001 \\
\hline Coronary heart disease $-/+$ & $20 / 0$ & $25 / 4$ & $27 / 7$ & A: 0.035; B: 0.008; C: NS \\
\hline Hypertension $-/+$ & $20 / 0$ & $25 / 4$ & $21 / 13$ & A: $0.035 ; B:<0.001 ; C: 0.026$ \\
\hline Smokers -/+ & $17 / 3$ & $16 / 13$ & $11 / 23$ & A: $0.024 ; B:<0.001 ; C: N S$ \\
\hline Diabetes mellitus -/+ & $20 / 0$ & $29 / 0$ & $24 / 10$ & A: NS; B: 0.001; C: 0.001 \\
\hline Metabolic syndrome -/+ & $20 / 0$ & $23 / 6$ & $11 / 23$ & A: $0.03 ; B:<0.001 ; C:<0.001$ \\
\hline Total cholesterol [mmol/l] & $198(38)$ & $326(57)$ & $268(34)$ & $\mathrm{A}:<0.001 ; \mathrm{B}:<0.001 ; \mathrm{C}:<0.001$ \\
\hline High density lipoprotein [mmol/l] & $55(19)$ & $39(19)$ & $39(10)$ & A: $0.027 ; B: 0.001 ; C: N S$ \\
\hline Low density lipoprotein (mmol/l) & $125(65)$ & $243(36)$ & $186(52)$ & $\mathrm{A}:<0.001 ; \mathrm{B}:<0.001 ; \mathrm{C}:<0.001$ \\
\hline Apolipoprotein A [mg/dl] & $159(49)$ & $139(45)$ & $138(28)$ & A: NS; B: 0.021; C: NS \\
\hline Apolipoprotein B [mg/dl] & $112(35)$ & $176(60)$ & $147(37)$ & $\mathrm{A}:<0.001 ; \mathrm{B}:<0.001 ; \mathrm{C}: 0.002$ \\
\hline Lipoprotein (a) [mg/dl] & $13(20)$ & $15(14)$ & $11(18)$ & A: NS; B: NS ; C: NS \\
\hline Glucose $[\mathrm{mmol} / \mathrm{l}]$ & $88(11)$ & $91(13)$ & $93(29)$ & A: NS; B: NS ; C: NS \\
\hline Insulin $[\mu \mathrm{U} / \mathrm{ml}]$ & $7(8)$ & $7(4)$ & $10(7)$ & A: NS; B:0.04; C: NS \\
\hline HOMA-IR & $1.4(2)$ & $1.7(0.7)$ & $2.4(2.1)$ & A: NS; B: 0.014; C:0.04 \\
\hline $\mathrm{TG}_{0}[\mathrm{mmol} / \mathrm{l}]$ & $87(49)$ & $134(90)$ & $234(180)$ & A: $0.002 ; B:<0.001 ; C: 0.001$ \\
\hline $\mathrm{TG}_{2}[\mathrm{mmol} / \mathrm{l}]$ & $120(75)$ & $186(156)$ & 325 (176) & A: $0.003 ; B:<0.001 ; C:<0.001$ \\
\hline $\mathrm{TG}_{4}[\mathrm{mmol} / \mathrm{l}]$ & $139(79)$ & $208(132)$ & $401(241)$ & A: $0.002 ; B:<0.001 ; C:<0.001$ \\
\hline $\mathrm{TG}_{6}[\mathrm{mmol} / \mathrm{l}]$ & $124(96)$ & $230(156)$ & $359(217)$ & A: $0.001 ; B:<0.001 ; C: 0.001$ \\
\hline $\mathrm{TG}_{8}[\mathrm{mmol} / \mathrm{l}]$ & $97(77)$ & $187(122)$ & 317 (170) & A: 0.007; B: <0.001; C: $<0.001$ \\
\hline TG AUC $[\mathrm{mmol} / \mathrm{l} / \mathrm{h}]$ & $1011(652)$ & $1503(1147)$ & $2678(1415)$ & A: 0.001; B: $<0.001 ; C:<0.001$ \\
\hline
\end{tabular}

All values are presented as median (IQR) or percentages for categorical variables. $T G_{0}$ - fasting plasma triglyceride concentration. $T G_{2}, T G_{4}, T G_{6}$, $T G_{8}$ - plasma triglyceride concentration 2, 4, 6, 8 hours after the fat load, respectively. TG AUC - area under the curve for triglycerides HOMA-IR - index of homeostasis model of insulin resistance, $N S$ - not statistically significant

$A-$ controls vs. $F H, B-$ controls vs. $F C H, C-F H$ vs. $F C H$

a group of healthy subjects. We showed that FCH subjects demonstrate significant postprandial hypertriglyceridaemia, compared to $\mathrm{FH}$ and healthy subjects. In addition, $\mathrm{FCH}$ subjects with hypertriglyceridaemic (IV) and mixed (IIB) phenotypes show an exaggerated response to a fatty meal, compared to $\mathrm{FCH}$ subjects with hypercholesterolaemic (IIA) phenotype. On the other hand, postprandial lipaemia is more pronounced in hypercholesterolaemic $\mathrm{FCH}$, compared to hypercholesterolaemic $\mathrm{FH}$.

The prevalence of metabolic syndrome (defined by ATPIII) [14] among FCH subjects was $68 \%$, which is similar to that reported by others [19]. In line with previous studies, postprandial hypertriglyceridaemia was more significant in $\mathrm{FH}$ patients, compared to healthy subjects [18] and $\mathrm{TG}_{0}$ levels were positively correlated to the amount of postprandial lipaemia, as expressed by TG-AUC $[15,18,20-22]$. We previously showed that $\mathrm{FH}$ patients with an abnormal TG response had higher baseline TG levels, compared to $\mathrm{FH}$ with a normal response [18]. Therefore, in the current study we did not assess the differences in the postprandial response between $\mathrm{FH}$ patients with hypercholesterolaemic and mixed phenotypes. Conflicting opinions exist regarding the role of $L D L$ receptors (LDLr) in chylomicron clearance in $\mathrm{FH}$ patients [23-25]. Nevertheless, delayed chylomicron clearance, secondary to LDLr deficiency, is considered as one of the possible mechanisms responsible for the abnormal postprandial response in some $\mathrm{FH}$ subjects [26]. Moreover, coexistence of mutations in the LDLr related protein (LRP) has been linked to LDLr mRNA downregulation, in the postprandial state [27].

An abnormal response to fat loading in $\mathrm{FCH}$ patients has already been demonstrated by other 
Table II. Clinical characteristics of different subgroups according to lipoprotein phenotype

\begin{tabular}{|c|c|c|c|c|c|}
\hline Characteristics & $\mathrm{FH} \| \mathrm{A}(n=21)$ & $\mathrm{FH} I I \mathrm{~B}(n=8)$ & $\mathrm{FCH} \| \mathrm{A}(n=13)$ & $\mathrm{FCH} \| \mathrm{IB}(n=10)$ & $\mathrm{FCH}$ IV $(n=11)$ \\
\hline Age [years] & $37(12)^{\neq}$ & $37(12)$ & $49(15)^{\neq}$ & $45(8)$ & $48(9)$ \\
\hline $\mathrm{BMI}\left[\mathrm{kg} / \mathrm{m}^{2}\right]$ & $25(2)^{\neq}$ & $26(3)$ & $28(3)^{\neq}$ & $30(5)$ & $30(4)$ \\
\hline Waist (cm) & $92(7)^{\neq}$ & $98(9)$ & $99(9) \neq$ & $107(11)$ & $103(10)$ \\
\hline Coronary heart disease $-/+$ & $18 / 3$ & $7 / 1$ & $8 / 5 \S$ & $10 / 0 \S$ & $9 / 2$ \\
\hline Hypertension $-/+$ & $20 / 1^{\neq}$ & $5 / 3$ & $7 / 6^{ \pm}$ & $7 / 3$ & $7 / 4$ \\
\hline Smokers $-/+$ & $14 / 7$ & $2 / 6$ & $6 / 7$ & $3 / 7$ & $2 / 9$ \\
\hline Diabetes mellitus - & $21 / 0^{\neq}$ & $8 / 0$ & $10 / 3 \neq$ & $8 / 2$ & $6 / 5$ \\
\hline Metabolic syndrome $-/+$ & $19 / 2^{\neq}$ & $4 / 4$ & $5 / 8^{\neq}$ & $5 / 5 \mathrm{~A}$ & $1 / 10^{A}$ \\
\hline Total cholesterol [mmol/l] & $338(47)^{\neq}$ & $360(59)^{\dagger}$ & $273(14)^{*} \neq$ & $277(25)^{\dagger A}$ & $239(42)^{\star} A_{A}$ \\
\hline High density lipoprotein [mmol/l] & $47(15)$ & $40(9)$ & $43(11)$ & $37(8)$ & $38(6)$ \\
\hline Low density lipoprotein [mmol/l] & $258(48) \neq$ & $253(47)^{\dagger}$ & $204(18)^{\S^{*} \neq}$ & $185(23)^{\S+A}$ & $138(57)^{\star A}$ \\
\hline Apolipoprotein A [mg/dl] & $144(34)$ & $138(26)$ & $142(26)$ & $133(20)$ & $140(19)$ \\
\hline Apolipoprotein B [mg/dl] & $176(54)$ & $189(48)$ & $152(28)$ & $160(16)$ & $155(45)$ \\
\hline Lipoprotein (a) [mg/dl] & $23(24)$ & $18(10)$ & $21(16)$ & $10(6)$ & $28(41)$ \\
\hline Glucose $[\mathrm{mg} / \mathrm{dl}]$ & $90(8)$ & $92(7)$ & $105(38)$ & $120(42)$ & $122(68)$ \\
\hline Insulin $[\mu \mathrm{U} / \mathrm{ml}]$ & $7(4)$ & $8.5(3)$ & $8(5)$ & $14(8)$ & $12(7)$ \\
\hline HOMA-IR & $1.7(0.9)$ & $2.6(1.8)$ & $1.9(1.1)^{\S}$ & $4.4(2.6) \S$ & $4.4(4.5)$ \\
\hline $\mathrm{TG}_{0}[\mathrm{mmol} / \mathrm{l}]$ & $110(32)$ & $238(44)$ & $124(33)^{\S^{*}}$ & $276(51)^{\S}$ & $333(125)^{\star}$ \\
\hline $\mathrm{TG}_{2}[\mathrm{mmol} / \mathrm{l}]$ & $168(61)^{\neq}$ & $310(79)$ & $225(86)^{\S^{*} \neq}$ & $413(111)^{\S}$ & $377(108)^{\star}$ \\
\hline $\mathrm{TG}_{4}[\mathrm{mmol} / \mathrm{l}]$ & $203(73)$ & 391(107) & $270(103)^{\S}$ & $520(145)^{\S}$ & $434(121)^{\star}$ \\
\hline $\mathrm{TG}_{6}[\mathrm{mmol} / \mathrm{l}]$ & $198(77)^{\neq}$ & $457(142)$ & $276(104)^{\S * \neq}$ & $478(117)^{\S}$ & $442(129)^{\star}$ \\
\hline $\mathrm{TG}_{8}[\mathrm{mmol} / \mathrm{l}]$ & $146(60) \neq$ & 346 (144) & $204(67) \S^{\star *} \neq$ & $373(61)^{\S}$ & $383(97)^{\star}$ \\
\hline TG AUC $[\mathrm{mmol} / \mathrm{l} / \mathrm{h}]$ & $1374428)^{\neq}$ & $2992(660)$ & $1863(577) \S^{\S^{*} \neq}$ & $3409(770)^{\S}$ & $3220(824)^{*}$ \\
\hline
\end{tabular}

All values are presented as mean $\pm S D$ or percentages for categorical variables. $T G_{0}$ - fasting plasma triglyceride concentration, $T G_{2}, T G_{4}, T G_{6}$, $T G_{8}$ - plasma triglyceride concentration 2, 4, 6, 8 hours after the fat load, respectively, TG AUC-area under the curve for triglycerides, HOMA$I R$-index of homeostasis model of insulin resistance, NS - not statistically significant

* Significantly different between groups FCH $\| A$ and FH IIA $(p \leq 0.05)$, † Significantly different between groups FCH IIB and FH IIB (p $\leq 0.05)$, $\S$ Significantly different between groups FCH IIA and FCH IIB $(p \leq 0.05)$, * Significantly different between groups FCH IIA and FCH IV ( $p \leq 0.05)$, A Significantly different between groups FCH IIB and FCH IV $(p \leq 0.05)$

investigators; however, the number of patients in these studies was relatively small [28-29]. In our study, TG-AUC was $264 \%$ higher compared to the control group, which is lower than that found by others [28]. In addition, peak TG levels (401 \pm 241 $\mathrm{mg} / \mathrm{dl}$ ) were seen at $4 \mathrm{~h}$ following the fat loading test and were lower than peak TG levels found by others $[28,29]$. The above findings could be partially attributed to the different amount of fat load that was used by other investigators. Based on our previous studies, we defined an increased TG response to the fatty meal as any postprandial TG concentration higher than the highest TG concentration $(220 \mathrm{mg} / \mathrm{dl})$ observed in healthy subjects [30].

To our knowledge, this is the first study to compare postprandial lipaemia between $\mathrm{FH}$ and
$\mathrm{FCH}$. The $\mathrm{FCH}$ group demonstrated higher $\mathrm{TG}_{0}$ levels, abnormal postprandial lipaemia and delayed TG clearance, compared to $\mathrm{FH}$. Hypercholesterolaemic FCH patients (IIA) showed significantly higher postprandial TG, in comparison to $\mathrm{FH}$ patients with the same Fredrickson phenotype, despite the fact that $\mathrm{TG}_{0}$ concentrations were normal in both groups and did not differ significantly. There are two possible explanations for this. Firstly, FCH-IIA patients were older than FHIIA (mean age 49[15] vs. 37[12]; $p=0.01$ ). Other studies have shown that the postprandial TG response becomes impaired with aging [31]. Secondly, hypertension, DM and central obesity (as expressed by waist circumference) were more prevalent in the $\mathrm{FCH}$-IIA group ( $p=0.004, p=0.02$ and $p=0.03$, respectively). The prevalence of 


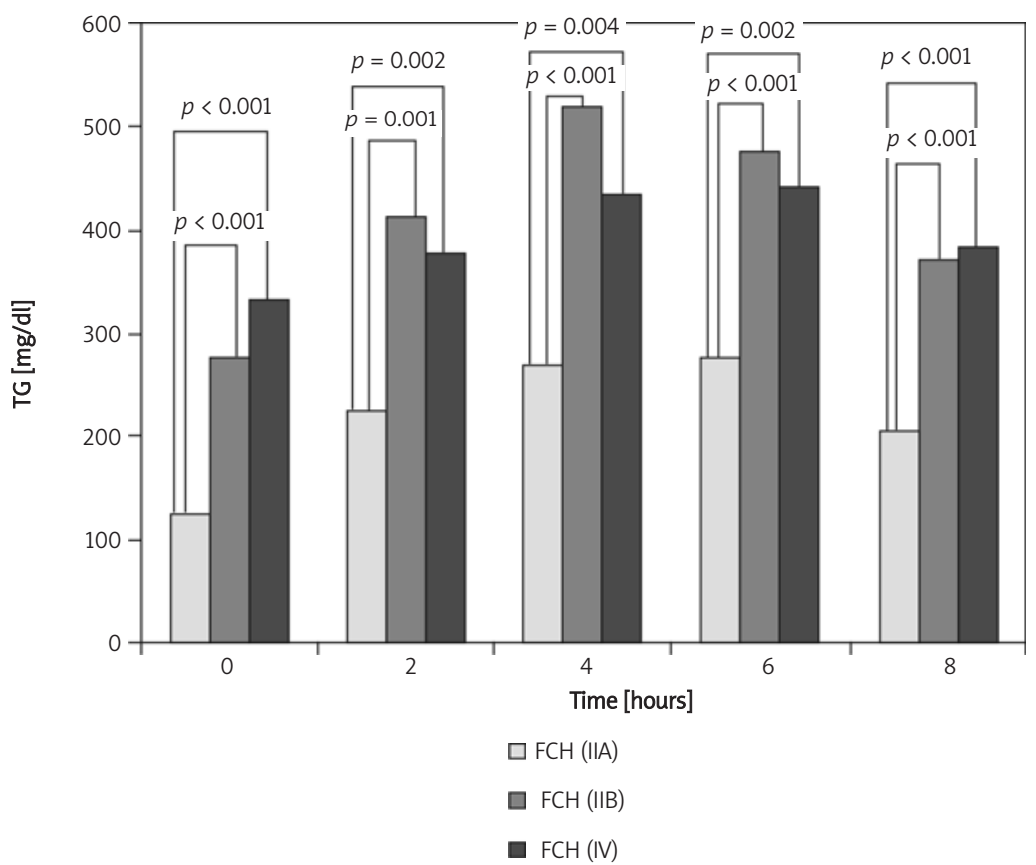

Figure 1. Comparison of TG concentrations at 0, 2, 4, 6, 8 hours between the three different familial combined hyperlipidaemia (FCH) subgroups, according to Fredrickson lipoprotein phenotype

metabolic syndrome among FCH-IIA and FH-IIA patients was $62 \%$ and $10 \%$ respectively. Our previous study clearly showed that male patients with metabolic syndrome demonstrate abnormal TG clearance following a fat loading test, even when $\mathrm{TG}_{0}$ levels are within normal limits [20]. Hypertension and DM have also been linked to abnormal postprandial lipaemia [15, 32].

On the other hand, although the $\mathrm{FCH}$-IIB subgroup demonstrated higher fasting and postprandial TG levels, compared to FCH-IV, these differences never reached statistical significance. Parameters that can affect postprandial clearance, such as DM, hypertension, metabolic syndrome and obesity, did not differ among the two subgroups, while $\mathrm{TG}_{0}$ levels were comparable. An important finding of the present study is the fact that phenotypes FCH-IIB and FCH-IV, which showed a greater postprandial response, had lower LDL levels, but higher levels of glucose and insulin (although the differences were not statistically significant), with high prevalence of diabetes. Diabetic FCH patients were compared to nondiabetic FCH patients and no additional exacerbation of postprandial lipaemia was revealed (data not shown). Insulin resistance has been linked to abnormal postprandial response [20-23, 33]. It is associated with fasting hypertriglyceridaemia [34], downregulation of LDLr [35] and overproduction of VLDL particles $[36,37]$. These effects increase competition between chylomicron and VLDL remnants for hepatic receptors, thereby impairing the uptake of CM remnants by this pathway [38]. It is also known that in dyslipidaemias with IIB and IV phenotypes, where insulin resistance is usually common, cholesteryl ester transfer protein (CETP) acts as a mediator for the production of atherogenic small dense LDL particles [39].

Assessing the postprandial response in different phenotypic expressions of $\mathrm{FCH}$ can be of great clinical importance, in view of the intra-individual variability of the lipid profile. Phenotypic alterations, in the same subject, can cause a variable postprandial TG clearance in different periods of time, and therefore periodic adjustment of hypolipidaemic treatment may be required.

Limitations of our study include the small number of patients in the different subgroups and the non-age-matched comparisons between the main groups.

In summary, $\mathrm{FH}$ and $\mathrm{FCH}$ subgroups with hypertriglyceridaemic and mixed phenotypes demonstrate a significantly abnormal postprandial TG response. These subgroups of patients should be identified early and receive appropriate hypolipidaemic treatment, as they may be at higher risk of developing early atherosclerosis. This is especially a challenge in $\mathrm{FCH}$ patients who show intra-individual variability of their lipid profile and possibly alter their postprandial metabolic status, 
within a certain period of time. Fibrates or statins with a potent effect on TG or combination therapy with statins plus fibrates or statins plus niacin could be considered as the appropriate treatment, according to the fasting phenotype.

\section{Acknowledgements}

We are grateful to Alexandra Valaora, a dietitian, for expertly managing the patients during the meal.

\section{References}

1. Ingelsson E, Pencina MJ, Tofler GH, et al. Multimarker approach to evaluate the incidence of the metabolic syndrome and longitudinal changes in metabolic risk factors: the Framingham Offspring Study. Circulation 2007; 116: 984-92.

2. Assmann G, Schulte H. Relation of high-density lipoprotein cholesterol and triglycerides to incidence of atherosclerotic coronary artery disease (the PROCAM experience). Prospective Cardiovascular Münster study. Am J Cardiol 1992; 70: 733-7.

3. The multiple risk factor intervention trial (MRFIT). A national study of primary prevention of coronary heart disease. JAMA 1976; 235: 825-7.

4. Mora S, Rifai N, Buring JE, Ridker PM. Fasting compared with nonfasting lipids and apolipoproteins for predicting incident cardiovascular events. Circulation 2008; 118: 993-1001.

5. Kolovou GD, Anagnostopoulou KK, Daskalopoulou SS, Mikhailidis DP, Cokkinos DV. Clinical relevance of postprandial lipaemia. Curr Med Chem 2005; 12: 1931-45.

6. Patsch JR, Miesenbock G, Hopferwieser T, et al. Relation of triglyceride metabolism and coronary artery disease. Studies in the postprandial state. Arterioscler Thromb 1992; 12: 1336-45.

7. Gaddi A, Galetti C, Pauciullo P, Arca M. Familial combined hyperlipoproteinemia: experts panel position on diagnostic criteria for clinical practice. Committee of experts of the Atherosclerosis and Dysmetabolic Disorders Study Group. Nutr Metab Cardiovasc Dis 1999; 9: 304-11.

8. Goldstein JL, Schrott HG, Hazzard WR, Bierman EL, Motulsky AG. Hyperlipidemia in coronary heart disease. II. Genetic analysis of lipid levels in 176 families and delineation of a new inherited disorder, combined hyperlipidemia. J Clin Invest 1973; 52: 1544-68.

9. Scientific Steering Committee on behalf of the Simon Broome Register Group. Risk of fatal coronary heart disease in familial hypercholesterolaemia. BMJ 1991; 303: 893-6.

10. Veerkamp MJ, de Graaf J, Hendriks JC, Demacker PN, Stalenhoef AF. Nomogram to diagnose familial combined hyperlipidemia on the basis of results of a 5-year followup study. Circulation 2004; 109: 2980-5.

11. Gaddi A, Cicero A, Odoo F, Poli AA, Paoletti R. Atherosclerosis and Metabolic Diseases Study Group. Practical guidelines for familial combined hyperlipidemia diagnosis: an up-date. Vasc Health Risk Manag 2007; 3: 877-86.

12. Rifkind BM, Segal P. Lipid Research Clinics Program reference values for hyperlipidemia and hypolipidemia. JAMA 1983; 250: 1869-72.

13. Kolovou GD, Anagnostopoulou KK, Pilatis ND, et al. The influence of natural menopause on postprandial lipemia in heterozygotes for familial hypercholesterolemia. J Womens Health 2004; 13: 1119-26.

14. Expert Panel on Detection, Evaluation, And Treatment of High Blood Cholesterol in Adults (Adult Treatment Panel III). Executive Summary of the Third Report of the National Cholesterol Education Program (NCEP) Expert Panel on Detection, Evaluation, and Treatment of High Blood Cholesterol in Adults (Adult Treatment Panel III). JAMA 2001; 285: 2486-97.

15. Kolovou GD, Daskalova DCh, Iraklianou SA, et al. Postprandial lipemia in hypertension. J Am Coll Nutr 2003; 22: 80-7.

16. Friedewald WT, Levy RI, Fredrickson DS. Estimation of concentrations of low density lipoprotein cholesterol in plasma without use of preparative ultracentrifuge. Clin Chem 1972; 18: 499-502.

17. Matthews DR, Hosker JP, Rudenski AS, Naylor BA, Treacher DF, Turner RC. Homeostasis model assessment: insulin resistance and beta-cell function from fasting plasma glucose and insulin concentrations in man. Diabetologia 1985; 28: 412-9.

18. Kolovou GD, Anagnostopoulou KK, Pilatis ND, et al. Heterozygote Men with Familial Hypercholesterolaemia may have an Abnormal Triglyceride Response postprandially. Evidence for another predictor of vascular risk in Familial Hypercholesterolaemia. Int J Clin Pract 2005; 59: 311-7.

19. Hopkins PN, Heiss G, Ellison RC, et al. Coronary artery disease risk in familial combined hyperlipidemia and familial hypertriglyceridemia: a case-control comparison from the National Heart, Lung, and Blood Institute Family Heart Study. Circulation 2003; 108: 519-23.

20. Kolovou GD, Anagnostopoulou KK, Pavlidis AN, et al. Postprandial Lipemia in Men with Metabolic Syndrome, Hypertensives and Healthy Subjects. Lipids Health Dis 2005; 4: 21.

21. Kolovou GD, Anagnostopoulou KK, Pavlidis AN, et al. Postprandial lipaemia in menopausal women with metabolic syndrome. Maturitas 2006; 55: 19-26.

22. Kolovou GD, Anagnostopoulou KK, Pavlidis AN, et al. Metabolic syndrome and gender differences in postprandial lipaemia. Eur J Cardiovasc Prev Rehabil 2006; 13: 661-4.

23. Field PA, Gibbons GF. Decreased hepatic expression of the low-density lipoprotein (LDL) receptor and LDL receptorrelated protein in aging rats is associated with delayed clearance of chylomicrons from the circulation. Metabolism 2000; 49: 492-8.

24. Mortimer BC, Beveridge DJ, Phan CT, Lutton C, Redgrave TG. The diurnal rhythms of cholesterol metabolism and plasma clearance of model chylomicrons: comparison of normal and genetically hypercholesterolemic rats (RICO). Comp Biochem Physiol A Mol Integr Physiol 1998; 120: 671-80.

25. Kowal RC, Herz J, Weisgraber KH, Mahley RW, Brown MS, Goldstein JL. Opposing effects of apolipoproteins E and C on lipoprotein binding to low density lipoprotein receptorrelated protein. J Biol Chem 1990; 265: 10771-9.

26. Watts GF. Postprandial lipaemia in familial hypercholesterolaemia: clinical and metabolic significance. Atherosclerosis 2000; 148: 426-8.

27. Pocathikorn A, Taylor RR, James I, Mamotte CD. LDLreceptor mRNA expression in men is downregulated within an hour of an acute fat load and is influenced by genetic polymorphism. J Nutr 2007; 137: 2062-7. 
28. Meijssen S, Cabezas MC, Twickler TB, Jansen H, Erkelens DW. In vivo evidence of defective postprandial and postabsorptive free fatty acid metabolism in familial combined hyperlipidemia. J Lipid Res 2000; 41: 1096-102.

29. Castro Cabezas M, Verseyden C, Meijssen S, Jansen $H$, Erkelens DW. Effects of atorvastatin on the clearance of triglyceride-rich lipoproteins in familial combined hyperlipidemia. J Clin Endocrinol Metab 2004; 89: 5972-80.

30. Anagnostopoulou KK, Kolovou GD, Kostakou PM, et al. Sex-associated effect of CETP and LPL polymorphisms on postprandial lipids in familial hypercholesterolaemia. Lipids Health Dis 2009; 8: 24.

31. Kolovou G, Anagnostopoulou K, Kostakou P, et al. Association between the TaqIB polymorphism in the cholesteryl ester transfer protein gene locus and postprandial plasma lipoprotein levels in heterozygotes for familial hypercholesterolemia. Clin Chem Lab Med 2007; 45: 1190-8.

32. Ginsberg HN, Illingworth DR. Postprandial dyslipidemia: an atherogenic disorder common in patients with diabetes mellitus. Am J Cardiol 2001; 88: 9H-15H.

33. Blackburn P, Lamarche B, Couillard C, et al. Contribution of visceral adiposity to the exaggerated postprandial lipemia of men with impaired glucose tolerance. Diabetes Care 2003; 26: 3303-9.

34. Després JP, Moorjani S, Lupien PJ, Tremblay A, Nadeau A, Bouchard C. Regional distribution of body fat, plasma lipoproteins, and cardiovascular disease. Arteriosclerosis 1990; 10: 497-511.

35. Mazzone T, Foster D, Chait A. In vivo stimulation of lowdensity lipoprotein degradation by insulin. Diabetes 1984; 33: 333-8.

36. Filippatos T, Tsimihodimos V, Kostapanos M, et al. Small dense LDL cholesterol and apolipoproteins C-II and C-III in non-diabetic obese subjects with metabolic syndrome. Arch Med Sci 2008; 3: 263-9.

37. Tziomalos K, Athyros V, Karagiannis A, Mikhailidis DP. Apolipoproteins C-II and C-III and small dense low density lipoprotein: novel risk factors in metabolic syndrome? Arch Med Sci 2008; 3: 270-3.

38. Cooper AD: Hepatic uptake of chylomicron remnants. J Lipid Res 1997; 38: 2173-92.

39. Guerin M, Le Goff W, Lassel TS, et al. Atherogenic role of elevated CE transfer from HDL to VLDL(1) and dense LDL in type 2 diabetes: impact of the degree of triglyceridemia. Arterioscler Thromb Vasc Biol 2001; 21: 282-8. 